Marquette University

e-Publications@Marquette

Psychology Faculty Research and Publications

Psychology Department

$12-1-2010$

Memory for Emotionally Provocative Words in Alexithymia: A Role for Stimulus Relevance

Mitchell Meltzer

Marquette University

Kristy A. Nielson

Marquette University, kristy.nielson@marquette.edu

Post-print.

Consciousness and Cognition, Volume 19, No. 4 (December 2010), DOI: 10.1016/

j.concog.2010.05.008. 


\section{Memory for Emotionally Provocative Words in Alexithymia: A Role for Stimulus Relevance}

Authors: Mitchell A. Meltzer ${ }^{\mathrm{a}}$, Kristy A. Nielson ${ }^{\mathrm{a}, \mathrm{b}, *}$

Abstract: Alexithymia is associated with emotion processing deficits, particularly for negative emotional information. However, also common are a high prevalence of somatic symptoms and the perception of somatic sensations as distressing. Although little research has yet been conducted on memory in alexithymia, we hypothesized a paradoxical effect of alexithymia on memory. Specifically, recall of negative emotional words was expected to be reduced in alexithymia, while memory for illness words was expected to be enhanced in alexithymia.

Eighty-five high or low alexithymia participants viewed and rated arousing illness-related ("pain"), emotionally positive ("thrill"), negative ("hatred"), and neutral words ("horse"). Recall was assessed 45 min later.

High alexithymia participants recalled significantly fewer negative emotion words but also more illness-related words than low alexithymia participants. The results suggest that personal relevance can shape cognitive processing of stimuli, even to enhance retention of a subclass of stimuli whose retention is generally impaired in alexithymia.

\section{Introduction}

Alexithymia is a trait representing a cluster of characteristics that includes difficulty identifying, describing, and communicating feelings; difficulty distinguishing between the feelings and bodily sensations of arousal; constricted imaginal processes; and an externally oriented cognitive style (Berenbaum \& Irvin, 1996). Research has shown alexithymia to be a relatively stable trait under changing emotion, stress, and pathology levels (Luminet, Bagby, \& Taylor, 2001; Mikolajczak \& Luminet, 2006; Subic-Wrana, Bruder, Thomas, Lane, \& Kohle, 2005). Although its precise etiology is unknown, an association between alexithymia and emotional dysregulation has been postulated (Taylor, 1994). Thus, individuals with alexithymia tend to be less aware of and responsive to their emotional and arousal states, and they tend to frequently suffer from psychiatric and psychosomatic disorders (Taylor \& Bagby, 2004).

Studies on the cognitive effects of alexithymia are nearly nonexistent. However, studies of subjective evaluations of emotional stimuli in alexithymia suggest that alexithymic individuals appraise negative emotional stimuli as less arousing than do those who score low on alexithymia

1 Meltzer \& Nielson 
(Pollatos, Schubo, Herbert, Matthias, \& Schandry, 2008; Roedema \& Simons, 1999; Stone \& Nielson, 2001), although one study found that only implicit indices showed reduced appraisal of emotionally negative and body symptom words in alexithymia; explicit ratings did not differ in alexithymia (Mueller, Alpers, \& Reim, 2006). These findings collectively suggest reduced processing resource allocation specifically for negatively valenced information in alexithymia and reduced priming effects of negative stimuli (e.g., Vermeulen, Luminet, \& Corneille, 2006). Reduced subjective response to negative emotional stimuli has also been shown despite normal physiological responses to stimuli (Stone \& Nielson, 2001), supporting the contention that there is a "decoupling" of subjective and physiological arousal responses in alexithymia (Papciak, Feuerstein, \& Spiegel, 1985).

Few studies have specifically examined memory in alexithymia. One study examining only neutral words demonstrated poorer recall in high alexithymia (Nielson \& Meltzer, 2009). Memory for emotional words has produced more mixed results. One study using both positive and negative words found no effect of alexithymia on memory (Lundh, Johnsson, Sundqvist, \& Olsson, 2002). Two recent studies, however, demonstrated reduced retrieval in alexithymia. In the first study, high and low alexithymia participants, as measured on the Toronto Alexithymia Scale-20 (TAS-20; (Bagby, Parker, \& Taylor, 1994; Bagby, Taylor, \& Parker, 1994), viewed neutral, negative, and positive words while employing either perceptual processing (i.e., rating the font size) or semantic processing (i.e., judging definition accuracy) (Luminet, Vermeulen, Demaret, Taylor, \& Bagby, 2006). High alexithymia participants had reduced recall of both negative and positive emotion words when (a) they had been semantically processed and (b) when specific recollection (i.e., "remember" vs. "know") responses were considered. Similar effects were also reported in the second study, where positive and negative word recall was reduced in alexithymia, but only when the Difficulty Identifying Feelings (DIF) subscale of the TAS-20 was the measure of alexithymia, and "remember" recognition responses were reduced in alexithymia for positive and negative words when the DIF subscale or the Externally Oriented Thinking (EOT) subscale was the alexithymia measure (Vermeulen \& Luminet, 2009).

Importantly, a positive correlation is typically reported between amygdala activity and either negative or positive stimulus processing (Dannlowski et al., 2007; Garolera et al., 2007; Kugel et al., 2008). But in alexithymia, the correlation of amygdala activity with stimulus processing is negative, in particular for negative stimuli (Kugel et al., 2008). Because there is also evidence that amygdala activation is required for the typical preferential encoding (Gasic, Barco, Avila, \& Lerma, 2006) and consolidation of emotional stimuli (Kensinger \& Schacter, 
2006a; McGaugh, 2004), diminished memory for emotion words in alexithymia is not surprising. But these findings suggest that negative word memory could be most greatly influenced by alexithymia.

While alexithymic individuals may display impaired recall for emotion words, illness-related words, which have not yet been studied in this context, could yield enhanced retrieval. For example, increased Stroop interference has been shown in alexithymia for arousing taboo words relative to neutral words in a student sample (Parker, Taylor, \& Bagby, 1993) and for illness words relative to negative emotion words, but not relative to neutral words in a general adult sample (Lundh \& Simonsson-Sarnecki, 2002). Notably, taboo and illness words carry emotional charge without specific reference to negative emotions (Lundh \& Simonsson-Sarnecki, 2002), suggesting inherent processing differences for such stimuli attributable to alexithymia. However, one study contrasted with these findings. Observer-rated alexithymia in an inpatient psychosomatic treatment sample was associated with reduced emotional bias (i.e., Stroop interference) for both negative and bodily-symptom words, although explicit ratings did not differ (Mueller et al., 2006). Emotional bias for positive words did not differ by alexithymia. Self-rated alexithymia did not interact with emotional bias.

Alexithymia is associated with a high prevalence of functional somatic symptoms (Taylor, Parker, Bagby, \& Acklin, 1992), which may lead to a memory bias for illness words due to the personal relevance of these stimuli. Individuals with chronic disorders such as irritable bowel syndrome (IBS) and somatoform disorder (SFD) experience prevalent and distressing somatic symptoms and they exhibit memory biases toward material related to frequent experiences associated with those disorders (Gibbs-Gallagher et al., 2001; Lim \& Kim, 2005). Somatosensory amplification, or "the tendency to experience somatic sensations as intense, noxious, and disturbing" (Barsky, Goodson, Lane, \& Cleary, 1988, p. 510), has been found to positively correlate with levels of alexithymia in psychosomatic patients (Nakao, Barsky, Kumano, \& Kuboki, 2002), in patients with panic disorder (De Berardis et al., 2007), and in nonclinical samples (Nakao, Barsky, Nishikitani, Yano, \& Murata, 2007). Thus, the tendency in alexithymia to perceive somatic sensations, such as emotional arousal, as negative and idiopathic or due to illness (Bagby \& Taylor, 1997) may similarly contribute to memory biases.

To our knowledge, the present study is the first to examine memory biases for emotion and illness-related words in alexithymia using explicit retention tests. Eighty-five participants (59 females; mean age $=19.01, S D=1.41$ ) were categorized post-testing by a median split on the TAS-20 (Bagby, Parker, et al., 1994; Bagby, Taylor, et al., 1994) as either high alexithymia 
(TAS-20 > 42; mean $=50.40 ; S D=5.6 ; n=43$ ) or low (TAS-20 $\leq 42$; mean = $34.64(S D=5.5)$; $n=42$ ). The TAS-20 has good internal consistency in a college sample (Cronbach's $\alpha=.81$ ), good test-retest reliability $(r=.77)$, and good convergent and divergent validity (Bagby, Parker, et al., 1994; Bagby, Taylor, et al., 1994).

\subsection{Procedures and hypotheses}

Participants viewed 32 words from the Affective Norms for English Words database (Bradley \& Lang, 1999), which were chosen to represent four different categories: highly arousing positive emotion words, highly arousing negative emotion words, highly arousing illness-related words, and neutral common nouns (see Table 1). The words were presented in white lettering on blue background for six seconds [followed by a 10-s blank to allow for ratings (Bradley \& Lang, 1999)] in a quasi-randomized order designed to distribute categories equally throughout the list. Participants were asked to silently read each word and then rate it on valence (pleasant-unpleasant), arousal (excited-calm), and dominance (large-small) (Bradley \& Lang, 1999). No instructions were given to remember the words (i.e., incidental learning). Recall was unexpectedly tested after a 45-min interval of completing various surveys (including the TAS-20). It was predicted that high alexithymia participants would demonstrate poorer recall for highly arousing negative emotion words and greater recall for illness-related words than those low in alexithymia. It was also predicted that alexithymia would have no effect on valence ratings of words, but that it would result in reduced arousal ratings of illness and emotion words (Mueller et al., 2006; Pollatos et al., 2008; Roedema \& Simons, 1999; Stone \& Nielson, 2001).

\section{Results}

Overall, the alexithymia groups did not differ in total word recall, $t(83)=-1.27, p=.21$ (high mean $=33.7 \%, S D=9.1$; low mean $=31.5 \%, S D=6.5$ ), or total recall intrusions (i.e., words produced but which were not presented), $t(83)=0.26, p=.80$ (high mean $=2.26, S D=$ 1.75 ; low mean $=2.36, S D=1.85$ ).

\subsection{Word ratings}

Separate mixed ANOVAs (2-Group $\times$ 4-Word Type) for arousal, valence and dominance ratings of the words demonstrated the expected ANEW-norms pattern. However, there were no significant differences by or interactions with alexithymia group (see Fig. 1). Specifically, the main effect for Word Type was significant for each rating as expected (Valence $(\mathrm{V}): F(3,249)=$ 391.83, $p<.001, \eta_{p}^{2}=.825$; Arousal $(\mathrm{A}): F(3,243)=22.97, p<.001, \eta_{p}^{2}=.221$; Dominance (D): $\left.F(3,243)=71.25, p<.001, \eta_{p}^{2}=.468\right)$, but there were no significant Group main effects (V: $F(1$, 
83) $=0.04, p=.84, \eta_{p}^{2}=.000 ; \mathrm{A}: F(1,81)=.011, p=.92, \eta_{p}^{2}=.00 ; \mathrm{D}: F(1,81)=0.61, p=.44$, $\left.\eta_{p}^{2}=.007\right)$ or Word Type by Group interactions $\left(\mathrm{V}: F(3,249)=0.18, p=.91, \eta_{p}^{2}=.002 ; \mathrm{A}: F(3\right.$, 243) $\left.=1.48, p=.22, \eta_{p}^{2}=.018 ; \mathrm{D}: F(3,243)=0.84, p=.48, \eta_{p}^{2}=.010\right)$.

\subsection{Delayed free recall}

A 2-Group by 4-Word Type mixed ANOVA was used to analyze word recall. The main effect of Group was not significant, $F(1,83)=1.49, p=.225, \eta_{p}^{2}=.018$. However, there was a significant main effect of Word Type, $F(3,249)=18.23, p<.001, \eta_{p}^{2}=.180$, as well as a significant Group by Word Type interaction effect, $F(3,249)=6.78, p<.001, \eta_{p}^{2}=.076$. High

alexithymia participants recalled more illness words $\left(F(1,83)=12.8, p=.001, \eta_{p}^{2}=.134\right)$ and fewer negative emotion words $\left(F(1,83)=3.93, p=.05, \eta_{p}^{2}=.045\right)$ than did low alexithymia participants. There was also a trend toward greater recall of common nouns by high alexithymia participants $\left(F(1,83)=3.60, p=.06, \eta_{p}^{2}=.042\right)$. Positive emotion words did not differ between groups, $F(1,83)=0.58, p=.45, \eta_{p}^{2}=.007$. These results are depicted in Fig. 2.

Comparing within groups but between stimulus conditions, post hoc tests showed that illness words were less recalled than words from each other stimulus category (vs. negative ( $p$ $=.03)$, positive $(p<.001)$, neutral $(p<.001)$ in low alexithymia. Negative words were also less recalled than neutral words $(p=.009)$. In high alexithymia, illness words were more recalled than negative $(p=.001)$ and less recalled than neutral words $(p=.002)$. Negative words were also less recalled than either positive $(p=.006)$ or neutral words $(p<.001)$.

Word list recall correlations with TAS total score were generally consistent with the categorical analysis (IIIness words $r=.42(p<.001)$; Negative words $r=-.21(p=.05)$; Positive words $r=-.07(p=.50)$; Neutral words $r=.21(p=.05)$. Each of the three factor scores of the TAS also significantly correlated with illness word recall [Difficulty Identifying Feelings (DIF), $r$ $=.39$ ( $p<.001)$; Difficulty Describing Feelings (DDF), $r=.29(p<.007)$; Externally Oriented Thinking (EOT), $r=.30(p<.04)$ ], but not with recall on other lists or total word recall $(p=.12$ to .92) except for EOT, which trended toward a significant correlation with neutral word recall $(r$ $=.20, p=.065)$.

\section{Discussion}

As predicted, high alexithymia participants recalled significantly fewer negative emotion words than did those low in alexithymia. Deficits in both automatic and conscious processing of negative emotional stimuli have been noted in alexithymia (e.g., Mueller et al., 2006; Vermeulen 
et al., 2006). Alexithymia has also been associated with reduced amygdala activation in response to negative emotional stimuli (Kugel et al., 2008). Our results are therefore fitting with reduced processing of negative emotional stimuli in alexithymia. In contrast, although the results were in the same direction as in two previous studies (Luminet et al., 2006; Vermeulen \& Luminet, 2009), positive word recall did not significantly differ by alexithymia.

Also as predicted but not before studied, high alexithymia participants recalled significantly more illness-related words than did participants low in alexithymia. This finding is consistent with priming and Stroop studies showing increased interference and lexical decision-making time for illness-related stimuli in alexithymia (Lundh \& Simonsson-Sarnecki, 2002; Parker et al., 1993). For example, one study showed greater Stroop interference for illness words than negative words in high alexithymia, while there was no difference in low alexithymia (Lundh \& Simonsson-Sarnecki, 2002). Although one study contrasts with the current findings, its findings were specifically for observer-rated, but not self-rated alexithymia in an inpatient psychiatric sample (Mueller et al., 2006). The present study compares more directly with college or general population samples and self-rated alexithymia (Lundh \& Simonsson-Sarnecki, 2002; Parker et al., 1993). It should be noted that the illness words (and neutral words) were normatively more "concrete" than either positive or negative emotion words. As such, it is possible that illness words are easier to process in alexithymia because they are less abstract. Alternatively, it is possible that somatosensory amplification and somatic symptoms, which are common in alexithymia (De Berardis et al., 2007; Nakao et al., 2002, 2007; Taylor et al., 1992), led to the memory bias for illness words. That is, somatosensory amplification and somatic symptom prevalence may cause illness words to elicit enhanced cognitive processing due to personal relevance in alexithymia or they may elicit enhanced physiological arousal or amygdala responses in alexithymia (Kensinger \& Schacter, 2006a; McGaugh, 2004). These possibilities should be directly assessed and tested in future studies.

The trend towards enhanced recall of neutral words in high alexithymia participants was unanticipated and contrasts with the very limited prior literature (Luminet et al., 2006). However, a recent study found the EOT factor of the TAS-20 measure positively correlated with total word recall and neutral word recall specifically (Vermeulen \& Luminet, 2009). In the current study, EOT did not correlate with total recall, but it trended toward significance for neutral words. Additionally, imagery and concreteness ratings, which were not primarily considered for stimulus selection, showed that neutral words were more concrete and imageable than the other word categories. High imagery and concreteness values increase both encoding and retrieval efficacy

\section{Meltzer \& Nielson}


generally, which could be further enhanced in those with an EOT style. Thus, the present finding suggests that high alexithymia participants may have preferentially processed the neutral words because they were salient for those with externally oriented processing styles as the only class of non-emotive words in the task, or as the most concrete and imageable items.

The effects of emotional salience in alexithymia could also have caused better neutral word retrieval. One study demonstrated that emotional materials become more salient (and thus are better recollected) when emotional and neutral materials are combined. That is, separate lists demonstrate less emotional bias in retrieval than combined lists (Dewhurst \& Parry, 2000). However, in alexithymia, the difference in salience may not be evident for emotive materials, since emotive or arousing stimuli are generally evaluated with less subjective intensity (e.g., Pollatos et al., 2008; Roedema \& Simons, 1999; Stone \& Nielson, 2001) and evoke less priming and processing resource allocation (e.g., Vermeulen et al., 2006). In that event, emotive words lose their advantage, but neutral words of high concreteness and imagery quality could stand out. This interpretation also reinforces the contention that illness words have particular salience for alexithymics because despite their emotional tone, they are better recalled. These possibilities need further investigation.

Although ratings were in the predicted direction, the lack of significant group differences in arousal ratings was in contrast to our predictions and prior research (Mueller et al., 2006; Pollatos et al., 2008; Roedema \& Simons, 1999; Stone \& Nielson, 2001). However, important methodological differences existed between the present study and previous studies that may explain the differences. While the present study asked participants to view and rate words for arousal quality, previous studies used pictorial (Pollatos et al., 2008; Roedema \& Simons, 1999) or video stimuli (Stone \& Nielson, 2001). Emotional pictures tend to be more arousing than emotional words (Kensinger \& Schacter, 2006b). Perhaps differences in arousal ratings in alexithymia are more apparent with picture stimuli. Additionally, a median split was used to categorize alexithymia in the current study, while previous studies used more extreme comparisons [e.g., first vs. fifth quintiles on TAS-20 (Pollatos et al., 2008; Stone \& Nielson, 2001) or $>1 \mathrm{SD}$ above/below the mean (Roedema \& Simons, 1999)]. Although extremes were not needed to capture memory differences in alexithymia, perhaps these more extreme comparisons must be made to capture differences in arousal ratings in alexithymia.

The college sample used in the current study did not exhibit clinically significant alexithymia. The TAS- 20 scores ranged from 24 to 62 , indicating that the term "high alexithymia" used herein is relative to the "low alexithymia" group, but it does not imply alexithymia reached 
substantively into the clinical range. Two previous studies of recall of emotive words in alexithymia found reduced recall for both positive and negative emotion words in high alexithymia under at least certain task conditions (Luminet et al., 2006; Vermeulen \& Luminet, 2009). The lack of effect on positive words in the present study may indicate that alexithymia effects on positive word salience or retrieval may only be apparent when more extreme alexithymia is included. In addition, a 45-min delay occurred between learning and test in the current study, effectively testing long-term retention of incidentally learned words. Previous studies used relatively immediate retention tests. Thus, the effects of alexithymia on positive words may be apparent soon after learning, but may be short lived. This could possibly occur due to a form of the Pollyanna Principle where unconscious biases toward the positive may allow short-term reductions in retrieval due to alexithymia to be mitigated after longer retention intervals. Finally, it should be noted that emotionally enhanced memory in the general population is more typically seen with negative stimuli than with positive stimuli (e.g., Kern, Libkuman, Otani, \& Holmes, 2005), such that some have concluded that negative emotion is stronger than positive emotion in multiple domains (Rozin \& Royzman, 2001). As such, it is very possible that positive stimuli are simply less likely than negative stimuli to interact with alexithymia to alter memory. These possibilities should be investigated in future studies.

The results of this study offer important insights into the dynamics of verbal memory biases as well as into the nature of alexithymia. First, a memory bias toward illness-related words in alexithymia suggests that life experience and personal relevance shape the way in which we process and recall verbal information. Functional somatic symptoms were not measured in the current study, but in general, they occur at a high rate and are perceived as particularly distressing in alexithymia. Thus, personal relevance may have led to enhanced cognitive or neural processing of illness symptom words. Moreover, our findings suggest that the typical deficit in negative emotion processing often associated with alexithymia can be moderated by the personal relevance of emotional stimuli. Although all illness-related words were inherently negative, they do not refer to specific negative emotions, and thus contrast in recall with negative emotion words. Finally, these results may have implications for cognitive therapy when alexithymia is co-morbid. Specifically, certain therapeutic strategies, such as those aimed at reducing selective attention to negative environmental cues, may be ineffective or may need to be tailored for efficacy in those with alexithymia.

\section{Notes}

8 Meltzer \& Nielson 
- ${ }^{a}$ Department of Psychology and the Integrative Neuroscience Research Center, Marquette University, Milwaukee, WI 53201-1881, USA

- ${ }^{\mathrm{b}}$ Department of Neurology and the Center for Imaging Research, Medical College of Wisconsin, Milwaukee, WI, USA

- ${ }^{*}$ Corresponding author at: Department of Psychology, Marquette University, Milwaukee, WI 53201-1881, USA. Fax: +1 414288 5333. E-mail address: kristy.nielson@marquette.edu (K.A. Nielson).

\section{Acknowledgments}

- This work was supported in part by the Graduate School, Marquette University. A preliminary version of this study constituted a Master's Thesis (MAM).

\section{References}

Bagby, R. M., Parker, J. D. A., \& Taylor, G. J. (1994). The twenty-item Toronto Alexithymia Scale-I. Item selection and cross-validation of the factor structure. Journal of Psychosomatic Research, 38(1), 23-32.

Bagby, R. M., \& Taylor, G. J. (1997). Affect dysregulation and alexithymia. In G. J. Taylor, M. Bagby, \& J. D. A. Parker (Eds.), Disorders of affect regulation: Alexithymia in medical and psychiatric illness (pp. 26-45). Cambridge: Cambridge University Press.

Bagby, R. M., Taylor, G. J., \& Parker, J. D. A. (1994). The twenty-item Toronto Alexithymia Scale-II. Convergent, discriminant, and concurrent validity. Journal of Psychosomatic Research, 38(1), 33-40.

Barsky, A. J., Goodson, J. D., Lane, R. S., \& Cleary, P. D. (1988). The amplification of somatic symptoms. Psychosomatic Medicine, 50(5), 510-519.

Berenbaum, H., \& Irvin, S. (1996). Alexithymia, anger, and interpersonal behavior. Psychotherapy and Psychosomatics, 65(4), 203-208.

Bradley, M. M., \& Lang, P. J. (1999). Affective norms for English words (ANEW): Instruction manual and affective ratings (Technical Report C-1). Gainsville, FL: The Center for Research in Psychophysiology, University of Florida.

Cortese, M. J., \& Fugett, A. (2004). Imageability ratings for 3000 monosyllabic words. Behavior Research Methods, Instruments, \& Computers, 36(3), 384-387.

Dannlowski, U., Ohrmann, P., Bauer, J., Kugel, H., Arolt, V., Heindel, W., et al (2007). Amygdala reactivity predicts automatic negative evaluations for facial emotions. Psychiatry

9 Meltzer \& Nielson 
Research, 154(1), 13-20.

De Berardis, D., Campanella, D., Gambi, F., La Rovere, R., Sepede, G., Core, L., et al (2007).

Alexithymia, fear of bodily sensations, and somatosensory amplification in young outpatients with panic disorder. Psychosomatics, 48(3), 239-246.

Dewhurst, S. A., \& Parry, L. A. (2000). Emotionality, distinctiveness, and recollective experience. European Journal of Cognitive Psychology, 12, 541-551.

Garolera, M., Coppola, R., Munoz, K. E., Elvevag, B., Carver, F. W., Weinberger, D. R., et al (2007). Amygdala activation in affective priming: A magnetoencephalogram study. NeuroReport, 18(14), 1449-1453.

Gasic, G. P., Barco, A., Avila, J., \& Lerma, J. (2006). A meeting to remember: Meeting on memory and related disorders. EMBO Reports, 7(8), 768-773.

Gibbs-Gallagher, N., Palsson, O. S., Levy, R. L., Meyer, K., Drossman, D. A., \& Whitehead, W. E. (2001). Selective recall of gastrointestinal-sensation words: Evidence for a cognitive-behavioral contribution to irritable bowel syndrome. American Journal of Gastroenterology, 96(4), 1133-1138.

Kensinger, E. A., \& Schacter, D. L. (2006a). Amygdala activity is associated with the successful encoding of item, but not source, information for positive and negative stimuli. Journal of Neuroscience, 26(9), 2564-2570.

Kensinger, E. A., \& Schacter, D. L. (2006b). Processing emotional pictures and words: Effects of valence and arousal. Cognitive, Affective \& Behavioral Neuroscience, 6(2), 110-126.

Kern, R. P., Libkuman, T. M., Otani, H., \& Holmes, K. (2005). Emotional stimuli, divided attention, and memory. Emotion, 5(4), 408-417.

Kugel, H., Eichmann, M., Dannlowski, U., Ohrmann, P., Bauer, J., Arolt, V., et al (2008). Alexithymic features and automatic amygdala reactivity to facial emotion. Neuroscience Letters, 435(1), 40-44.

Lim, S. L., \& Kim, J. H. (2005). Cognitive processing of emotional information in depression, panic, and somatoform disorder. Journal of Abnormal Psychology, 114(1), 50-61.

Luminet, O., Bagby, R. M., \& Taylor, G. J. (2001). An evaluation of the absolute and relative stability of alexithymia in patients with major depression. Psychotherapy and Psychosomatics, 70(5), 254-260.

Luminet, O., Vermeulen, N., Demaret, C., Taylor, G. J., \& Bagby, R. M. (2006). Alexithymia and levels of processing: Evidence for an overall deficit in remembering emotion words. Journal of Research in Personality, 40(5), 713-733.

10 Meltzer \& Nielson 
Lundh, L.-G., Johnsson, A., Sundqvist, K., \& Olsson, H. (2002). Alexithymia, memory of emotion, emotional awareness, and perfectionism. Emotion, 2(4), 361-379.

Lundh, L.-G., \& Simonsson-Sarnecki, M. (2002). Alexithymia and cognitive bias for emotional information. Personality and Individual Differences, 32(6), 1063-1075.

McGaugh, J. L. (2004). The amygdala modulates the consolidation of memories of emotionally arousing experiences. Annual Review of Neuroscience, 27, 1-28.

Mikolajczak, M., \& Luminet, O. (2006). Is alexithymia affected by situational stress or is it a stable trait related to emotion regulation? Personality and Individual Differences, 40(7), 1399-1408.

Mueller, J., Alpers, G. W., \& Reim, N. (2006). Dissociation of rated emotional valence and Stroop interference in observer-rated alexithymia. Journal of Psychosomatic Research, 61(2), 261-269.

Nakao, M., Barsky, A. J., Kumano, H., \& Kuboki, T. (2002). Relationship between somatosensory amplification and alexithymia in a Japanese psychosomatic clinic. Psychosomatics, 43(1), 55-60.

Nakao, M., Barsky, A. J., Nishikitani, M., Yano, E., \& Murata, K. (2007). Somatosensory amplification and its relationship to somatosensory, auditory, and visual evoked and event-related potentials (P300). Neuroscience Letters, 415(2), 185-189.

Nielson, K. A., \& Meltzer, M. A. (2009). Modulation of long-term memory by arousal in alexithymia: The role of interpretation. Consciousness and Cognition, 18, 786-793.

Paivio, A., Yuille, J. C., \& Madigan, S. A. (1968). Concreteness, imagery, and meaningfulness values for 925 nouns. Journal of Experimental Psychology, 76(Suppl.), 1-25.

Papciak, A. S., Feuerstein, M., \& Spiegel, J. A. (1985). Stress reactivity in alexithymia: Decoupling of physiological and cognitive responses. Journal of Human Stress, 11(3), 135-142.

Parker, J. D. A., Taylor, G. J., \& Bagby, R. M. (1993). Alexithymia and the processing of emotional stimuli: An experimental study. New Trends in Experimental and Clinical Psychiatry, 9(1/2), 9-14.

Pollatos, O., Schubo, A., Herbert, B. M., Matthias, E., \& Schandry, R. (2008). Deficits in early emotional reactivity in alexithymia. Psychophysiology, 45(5), 839-846.

Roedema, T. M., \& Simons, R. F. (1999). Emotion-processing deficit in alexithymia. Psychophysiology, 36(3), 379-387.

Rozin, P., \& Royzman, E. B. (2001). Negativity bias, negativity dominance and contagion.

11 Meltzer \& Nielson 
Personality and Social Psychology Review, 5, 296-320.

Stone, L. A., \& Nielson, K. A. (2001). Intact physiological response to arousal with impaired emotional recognition in alexithymia. Psychotherapy and Psychosomatics, 70, 92-102.

Subic-Wrana, C., Bruder, S., Thomas, W., Lane, R. D., \& Kohle, K. (2005). Emotional awareness deficits in inpatients of a psychosomatic ward: A comparison of two different measures of alexithymia. Psychosomatic Medicine, 67(3), 483-489.

Taylor, G. J. (1994). The alexithymia construct: Conceptualization, validation, and relationship with basic dimensions of personality. New Trends in Experimental and Clinical Psychiatry, 10(2), 61-74.

Taylor, G. J., \& Bagby, R. M. (2004). New trends in alexithymia research. Psychotherapy and Psychosomatics, 73(2), 68-77.

Taylor, G. J., Parker, J. D. A., Bagby, R. M., \& Acklin, M. W. (1992). Alexithymia and somatic complaints in psychiatric out-patients. Journal of Psychosomatic Research, 36(5), 417-424.

Vermeulen, N., \& Luminet, O. (2009). Alexithymia factors and memory performances for neutral and emotional words. Personality and Individual Differences, 47, 305-309.

Vermeulen, N., Luminet, O., \& Corneille, O. (2006). Alexithymia and the automatic processing of affective information: Evidence from the affective priming paradigm. Cognition \& Emotion, 20(1), 64-91. 


\section{Appendix}

Table 1

Word lists by category with normative ratings and internal consistency of ratings made by participants in the study sample.

\begin{tabular}{|c|c|c|c|c|}
\hline & Illness & Negative & Positive & Neutral \\
\hline & $\begin{array}{l}\text { Agony } \\
\text { Burn } \\
\text { Pain } \\
\text { Poison } \\
\text { Rabies }^{\mathrm{e}} \\
\text { Stress }^{d} \\
\text { Suffocate }^{\text {Tumor }}\end{array}$ & $\begin{array}{l}\text { Afraid } \\
\text { Anger } \\
\text { Fear } \\
\text { Hatred } \\
\text { Rage }^{d} \\
\text { Rejected } \\
\text { Terrified } \\
\text { Violent }^{\mathrm{e}}\end{array}$ & $\begin{array}{l}\text { Affection } \\
\text { Desire }^{d} \\
\text { Fun } \\
\text { Happy } \\
\text { Joy } \\
\text { Love } \\
\text { Lust } \\
\text { Thrill }\end{array}$ & $\begin{array}{l}\text { Ankle } \\
\text { Door } \\
\text { Dress } \\
\text { Girl } \\
\text { Grass } \\
\text { Horse } \\
\text { Lake } \\
\text { Salad }\end{array}$ \\
\hline Valence $^{3}$ & $2.13(0.37) \alpha: 0.72$ & $2.13(0.41) \alpha: 0.76$ & $8.14(0.52) \propto: 0.79$ & $6.03(0.65) \alpha: 0.56$ \\
\hline Arousal $^{3}$ & $6.37(0.48) \alpha: 0.83$ & $7.15(0.65) \alpha: 0.84$ & $6.98(0.59) \alpha: 0.84$ & $4.01(0.18) \alpha: 0.57$ \\
\hline Dominance ${ }^{a}$ & $3.73(0.36) \alpha: 0.85$ & $4.20(1.15) \alpha: 0.81$ & $6.43(0.49) \propto: 0.79$ & $5.09(0.42) \alpha: 0.69$ \\
\hline Imagery $^{b, c}$ & $4.88(0.76)$ & $4.33(0.49)$ & $4.90(0.61)$ & $6.68(0.13)$ \\
\hline Concreteness ${ }^{b}$ & $4.29(0.84)$ & $2.66(0.81)$ & $2.35(0.65)$ & $6.92(0.07)$ \\
\hline
\end{tabular}

a, Cronbach $\alpha$.

a Bradley and Lang (1999).

${ }^{\mathrm{b}}$ Paivio, Yuille, and Madigan (1968).

${ }^{\mathrm{C}}$ Cortese and Fugett (2004).

${ }^{\mathrm{d}}$ Concreteness norms not available.

${ }^{\mathrm{e}}$ Imagery and concreteness norms not available. 


\section{Figure 1}
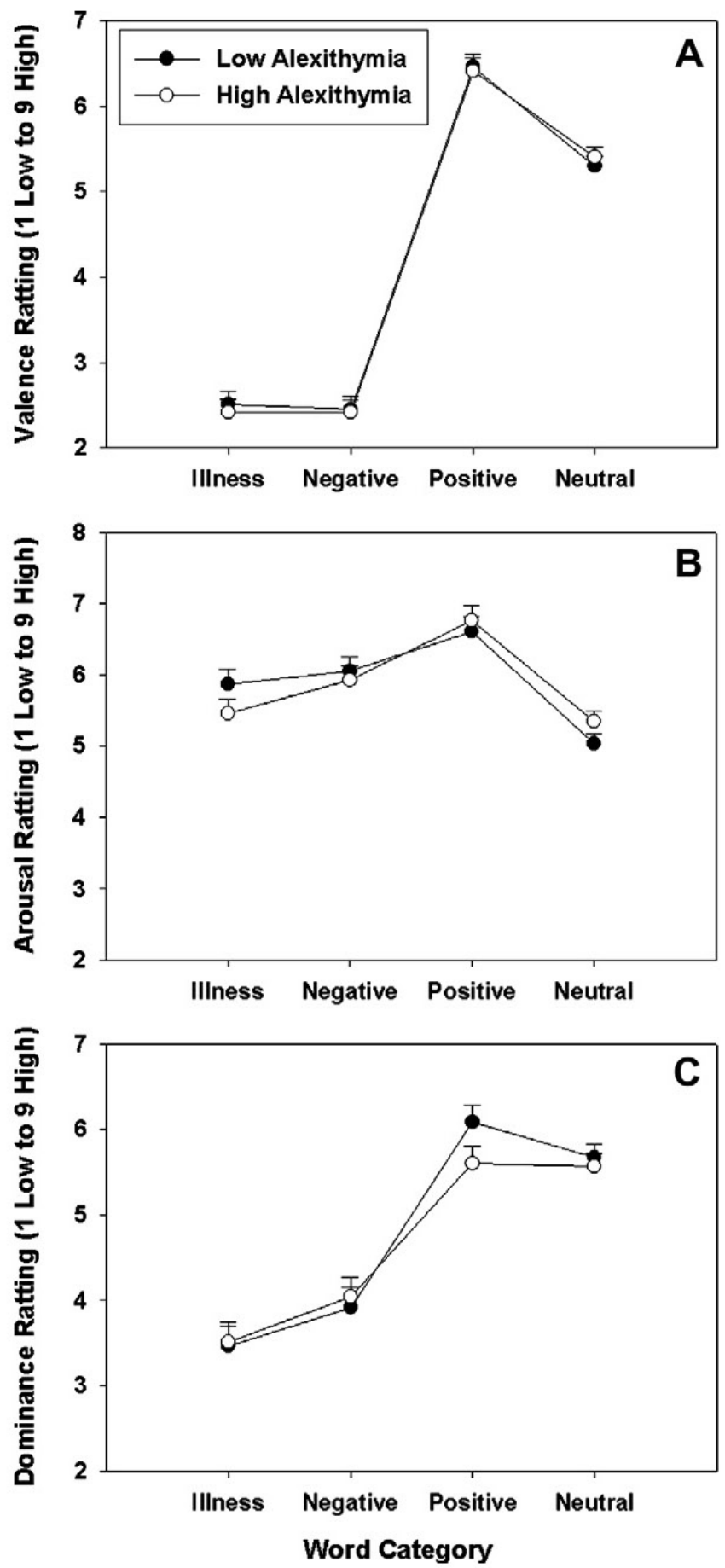

Low and high alexithymia group data (mean \pm SEM) are shown for subjective ratings of valence (pleasant/unpleasant, panel A), arousal (excited/calm, panel B), and dominance (controlled/in control, panel C) for each word stimulus category. There were no significant main effects or interactions involving alexithymia group.

\section{Meltzer \& Nielson}




\section{Figure 2}

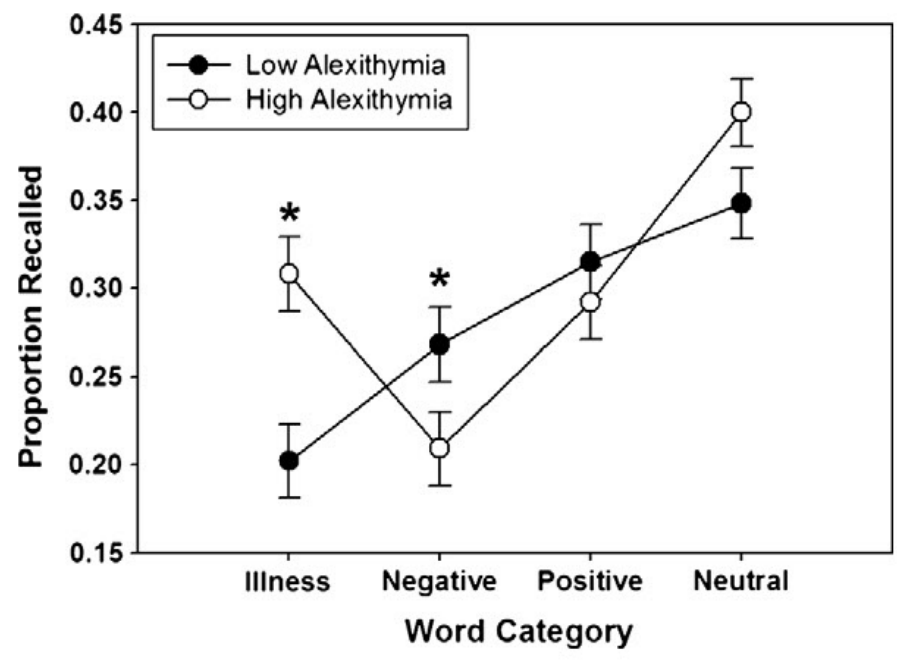

Word recall (mean \pm SEM) for each word stimulus category by alexithymia group is shown. The high alexithymia group recalled significantly more illness words and significantly fewer (non-illness-related) negative emotion words than did the low alexithymia group. High alexithymia participants trended toward better recall of neutral words as well $(p=.06)$. 